\title{
General Synthesis Techniques for Coupled Resonator Networks
}

\author{
Fabien Seyfert (INRIA, Sophia Antipolis, France), Stéphane Bila (Xlim, Limoges, France)
}

\begin{abstract}
With modern communication systems, the allocated frequency spectrum becomes more crowded and the demand for high-performance microwave filters complying with stringent specifications has considerably increased. Telecommunication systems require high-selectivity to prevent interference together with flat in-band groupdelay and amplitude to minimize signal degradation. The design of microwave filters is usually a tradeoff between various electrical performances (selectivity, insertion loss, group delay...) while minimizing mass and volume, development time and manufacturing cost [1]. For particular applications, additional constraints such as power-handling, thermal or mechanical stability have also to be analyzed carefully [2].

Several types and implementation technologies of distributed microwave filters [3] are available and the choice is driven by the application. However, the design is generally based on the same scheme [4]. The first step consists in synthesizing a lumped elements network from a polynomial filtering function that fulfills the electrical specifications. The second step then converts the lumped elements network into a practical microwave filter.

Applying this scheme, a designer has to face two major problems: the derivation of the lumped elements network which has to be compatible with the polynomial filtering function to be realized, and the dimensioning of the distributed microwave filter. This article details previous points, focusing on the design of coupled resonator filters, i.e. filters which comply with the coupling matrix representation.
\end{abstract}

\section{Compatibility of coupling topologies with specific classes of filtering functions}

As already mentioned in a preceding issue of this magazine [5, 6], the low pass prototype circuit (Figure 1) is widely used as a coarse model for the synthesis of coupled resonator filters. The coupling topology, or in other words, the way resonators are coupled to each other, is imposed by realisability issues that depend on the technology that is intended for the filter implementation. For example, in dual mode waveguide technology [7], the presence of diagonal cross couplings yields severe complications in the manufacturing process and efforts have been made to derive topologies that are "diagonal cross-coupling free" [8]. For planar technologies, elementary space constraints yield also some restrictions on the coupling topology and every designer faces inevitably the question: what kind of frequency responses can I possibly adjust given the constraints I have on my coupling topology? In the following, we give some guidelines to answer the latter.

The non-dissipative passive nature of the circuit (Figure 1) and its reciprocity $\left(S_{12}=S_{21}\right)$ implies mechanically the general polynomial form of its associated scattering matrix:

$$
S=\left[\begin{array}{ll}
S_{11} & S_{12} \\
S_{21} & S_{22}
\end{array}\right]=\frac{1}{E}\left[\begin{array}{cc}
F & P \\
P & (-1)^{n} F^{*}
\end{array}\right]
$$

where $n$ is the number of resonators and $F, P, E$ are polynomials with complex coefficients of the complex variable $s=\sigma+j \omega$ where ( $\omega$ is the normalized frequency).

The polynomial $P$ is of degree $m<n-1$ and satisfies the condition $P=(-1)^{n+1} P^{*}$ (which implies that the set of transmission zeros is symmetric with respect to the imaginary axis, i.e. paraconjugated). $F$ is of degree $n$ and monic, and the denominator $E$ is the unique Hurwitz polynomial satisfying following spectral equation:

$$
E E^{*}=F F^{*}+(-1)^{n+1} P^{2}
$$

Latter properties indicate that the scattering parameters are entirely governed by the two numerator polynomials $F$ and $P$ in terms of which the squared modulus of the transmission S-parameter expresses simply as:

$$
\left|S_{21}(j \omega)\right|^{2}=\frac{1}{1+\left|\frac{F(j \omega)}{P(j \omega)}\right|^{2}}
$$

where $D=F / P$ is known as the filtering or characteristic function.

Latter formula is the starting point of efficient frequency synthesis techniques and formulas exist, for example, for $F$ (given $P$ ) in order to obtain very selective quasi-elliptic filtering characteristics $[9,10]$. Techniques based 
on the predistortion of the filter response in order to compensate for the losses in the final device, make also heavy use of the general polynomial structure (1): in particular the reflexion zeros (zeros of $F$ ) are shifted inward the right complex plan by this method [11]. More recently, methods were developed to determine $F$ and $P$ in an optimal manner with respect to some general multi-band specifications [12]. In all these techniques, advantage is taken from the fact that $F$ and $P$ can be chosen freely up to limitations on their degrees and the paraconjugated nature of $P$. It is therefore natural to ask if these limitations are sufficient to ensure the realizability of a general polynomial scattering matrix of the form (1) by a low pass prototype circuit (Figure 1) with a specific coupling topology.

When no constraint is given on the topology of the coupling matrix, the answer to this question is yes and a constructive demonstration of this is given in [9]: in the latter, the author starts from a polynomial model and derives a full coupling matrix that realizes it (see also [13] for mathematical details). Reduction steps, involving the use of analytically computed similarity transforms (see [14]), allow reducing the full coupling matrix to matrices with well-known canonical topologies like the arrow form (Figure 2) and the folded form (Figure 3). In order to tackle more general coupling topologies, we first list necessary conditions relevant in the compatibility question between filtering characteristics and topologies.

Shortest path rule - For a given topology, let $l$ be the length of the shortest path in the coupling graph from the input to the output resonator. Then $n-l-1$ is the maximum number of transmissions zeros this topology can accommodate. This rule is an algebraic consequence of the structure of the low pass prototype and a proof of it can be found in [15].

Degrees of freedom of a class of filtering characteristics - For specific classes of filtering characteristics, we can evaluate the number of free parameters that define the polynomials $F$ and $P$. This number is called the dimension of the class. If $m$ is the number of allowed transmission zeros, we have:

- General asymmetric functions: $n$ complex transmission zeros can be chosen independently whilst $m+1$ real parameters define the polynomial $P$ (its coefficients are alternatively real and pure imaginary). This yields a total of $2 n+m+1$ free real parameters.

- Symmetric functions: for this kind of responses, $F$ has real coefficients and $P$ is restricted to be even (and therefore $m$ also). This yields a total of $n+m / 2+1$ free real parameters.

This little counting exercise leads to following useful rule: in order to accommodate a class of responses (ex. $(n, m)$ asymmetric) characterized by a given number of free parameters, a coupling topology must possess at least the same number of free electrical parameters. If these two numbers are equal then the realization problem has a finite number of solutions (but possibly none).

\section{Canonical coupling topologies: example of the arrow form}

The general arrow form entails following free electrical parameters: $n$ self couplings $M_{i, i}, n-1$ couplings between adjacent resonators $(M i, i+1), n-2$ additional couplings between the last resonator and all others, 2 source/load couplings which yield a total of $3 n-1$ free electrical parameters. Using the minimum path rule, the maximum number of transmission zeros is computed to be $(n-1)-2+1=n-2$. On the other hand, the number of free parameters for the $(n, n-2)$ asymmetric class is, according to our preceding remark, $2 n+n-2+1=3 n-1$, which is consistent with the fact that the arrow form is a canonical form as mentioned earlier. Moreover we may try here to give a precise definition of the intuitive notion of canonical form: if $\mathrm{C}$ is a class of responses of dimension $\mathrm{k}$, then a form is called canonical if it entails exactly $\mathrm{k}$ non zero independent electrical parameters and if the associated realization problem is guaranteed to have a single solution (up to the usual sign changes) for each element of $\mathrm{C}$.

Canonical forms adapted to responses with less transmission zeros can be obtained by enlarging the shortest path, i.e. by canceling progressively the $M_{k, n}$ couplings: the limiting form obtained by this procedure is the classical all pole topology, where resonators are coupled in a line. The latter is compatible with purely Chebyshev characteristics $((n, 0)$ type).

For symmetric characteristics, the use of topologies where all couplings $M_{i, j}$ are zero if $i+j$ is even are required: as a matter of fact the responses of such circuits are structurally symmetric $[14,16]$ so that no additional relations between couplings are necessary to ensure the symmetry of the response (i.e. the electrical parameters are free). This yields a general arrow form adapted to symmetric responses where all $M_{i, i}$ are set to 0 as well as every second coupling of the form $M_{k, n}$ : we leave to the reader's curiosity the care of verifying that the total number of free parameters in this form is equal to $n+(n-2) / 2+1$ (for $n$ even) which is also the dimension of the class of $(n, n-2)$ symmetric characteristics.

\section{General coupling topologies}

For general topologies, one may ask if our necessary conditions of compatibility between a topology and a class of functions are also sufficient: do they guaranty the existence of a solution to the coupling matrix synthesis problem? The answer to this question is roughly yes for the two classes we defined previously but additional 
material is needed (mathematical definition of non-redundancy) for a proper formulation: interested readers will find the complete statement of this compatibility condition in [16]. For practical matters, it is of course crucial to render latter statement constructive by deriving a general method that performs the realization step for filtering functions and topologies where the compatibility rules are fulfilled. The lack of an explicit reduction process for general topologies led the filtering community to derive various approaches based on optimization to solve the underlying non linear multivariate problem $[10,17]$. Even if latter algorithms perform relatively well in practice, no guaranty exists about the derivation of a solution, or all solutions, to the coupling matrix synthesis problem. A notable exception to this is made by [18] where a certified process is derived for special topologies made of cascaded triplets or quadruplets. Recently a procedure [16] based on the use of Groebner basis and homotopy techniques tackled the problem of solving exhaustively the related non linear system of equations and finally led to a complete solution of the synthesis problem for all relevant topologies (at least for the time being). This technique has been made accessible to the filtering community thru the software Dedale-HF [19], which is available on the web and free for any academic usage.

A typical application of this is made with the recently introduced extended box topologies [8] that are especially convenient for dual mode cavities filters with asymmetric characteristics. Consider for example the $8^{\text {th }}$ degree extended box topology in Figure 4. The shortest path rule indicates that at most 3 transmission zeros are supported by this topology. Counting the parameters yields 8 self-couplings, 10 couplings and 2 source/load couplings for a total of 20 free electrical parameters. On the other hand, the dimension of the class of $(8,3)$ asymmetric characteristics is, according to our formula, $2 \times 8+3+1=20$. The topology and the filtering characteristics class $(8,3)$ are therefore compatible (see [16] for a rigorous proof of this). Using Dedale-HF, a strongly asymmetric $(8,3)$ characteristic is computed (see Figure 5) and all 16 possible coupling matrices with the prescribed topology are derived. It is now up to the designer to decide which coupling matrix is most convenient for its application. For more details about this example, see Dedale-HF's tutorial [19] and [20] for applications to equivalent network simplification methods.

Another interesting class of characteristics are autoreciprocal ones, which are characterized by the additional condition $S 11=S 22$. Topologies that admit a symmetry plan across the centre of the circuit, i.e. that have a coupling matrix which is symmetric across both of its diagonals, are specially suited for this kind of responses as their scattering matrix is structurally autoreciprocal. Such topologies are called symmetric and our previous counting exercise can be repeated to derive necessary realisability conditions. For single band characteristics, the latter condition happens to be sufficient, but unfortunately, there exists auto-reciprocal dual band characteristics that admit no symmetric circuit realization. This technical point is beyond the scope of this paper but details on this will be given in forthcoming publications.

\section{Computer-aided design and tuning}

From the synthesized low-pass prototype circuit, normalized couplings can be used for a preliminary dimensioning of the distributed filter. This first-order dimensioning is generally not sufficient for a precise tuning, especially for narrow-band filters, even in presence of tuning element within the hardware. A more accurate dimensioning step, involving generally an electromagnetic model together with an elaborated process for tuning its dimensions, is then necessary. Moreover, computer-aided tuning is also necessary in some cases in order to guide the designer while adjusting the tuning elements (typically tuning screws) of a manufactured prototype.

Extracting coupling parameters from measured or simulated scattering data is an effective approach for tuning, step by step, an electromagnetic model or a hardware including tuning elements. Indeed, the comparison between identified parameters and synthesized ones provides an accurate diagnosis of tuning deviation and also, a direction for a better adjustment.

Pioneering works on computer-aided tuning of microwave filters [21] were based on optimizing the coupling parameters of an equivalent lumped element model by fitting the measured scattering parameters. However, the efficiency of such a straight approach depends on a favorable initial guess of the coupling parameters and substantial efforts have been spent up to now to propose more robust methods.

Currently, most of parameter extraction techniques [22- 25] consists in, firstly, deriving a rational approximation of the simulated or measured scattering parameters and, secondly, synthesizing the resulting low-pass coupled resonator network. A cornerstone of latter techniques is clearly the determination of a stable rational model of scattering parameters that coincides with the number of poles and zeros of the polynomial characteristic function [26]. The fundamental problem is to map the simulated or measured scattering parameters, which integrate delays due inherently to in/out coupling systems, with the polynomial formulation that is required for synthesizing the coupled resonator network. A strategy consists in estimating, then removing these delays by adjusting input/output reference planes $[26,27]$, in order to reduce the problem to a pure rational approximation problem. Figure 6 compares measured scattering parameters with their rational approximation.

Once a good rational approximation of the scattering parameters is found, the problem becomes once again to synthesize the low-pass coupled resonator network. In case of a coupled resonator network leading to a unique 
coupling matrix, for instance a canonical network, the synthesis always delivers a single coupling matrix that can be exploited for tuning iteratively the CAD model or the hardware. However, when several coupling matrices result from the synthesis, identifying the proper one is not always obvious, especially when the filter is substantially detuned.

A preliminary selection can then be completed by eliminating coupling matrices whose coupling signs are not consistent with the realized filter. Undeniably, coupling signs are controlled by the arrangement of coupling elements between resonators, and all coupling matrices which cannot correspond to this arrangement can be removed. A further step consists in tracking the evolution of remaining coupling matrices between close tuning steps. In this case, a tuning element is slightly modified in order to perturb the filter response and consequently the coupling matrices. Since the selected tuning element is related to a particular coupling parameter, the proper solution can be recognized by seeking for coherency between the tuning element modification and the evolution of coupling parameters within each coupling matrix. This step is done naturally while tuning the filter, but the number of tuning elements that are adjusted at the same time has to be limited in this case in order to follow the proper coupling matrix without ambiguity.

\section{Design Example}

The article is illustrated with the design of a 7 pole 3 zero dual-band band-pass filter. The two pass-bands are 50$\mathrm{MHz}$ wide and centered at $8.253 \mathrm{GHz}$ and $8.265 \mathrm{GHz}$ respectively. The generation of characteristic polynomials, from electrical specifications, is detailed in [12]. The resultant scattering parameters, normalized in the low-pass frequency domain, are shown in Figure 7.

The topology of the coupled resonator network that is chosen for realizing the previous characteristic, is a generalized extended-box topology presented in Figure 8. As can be verified using the guidelines of preceding section, this network is compatible with the class of $(7,3)$ asymmetrical characteristics. Using Dedale-HF, 3 possible realizations (4-6) of the ideal response are computed. Solution (4) is selected since having the most homogeneous coupling values.

$$
\left.\begin{array}{ccccccccc}
0 & 0.899 & 0 & 0 & 0 & 0 & 0 & 0 & 0 \\
0.899 & 0.076 & 0.265 & 0 & -0.823 & 0 & 0 & 0 & 0 \\
0 & 0.265 & -0.961 & 0.121 & 0 & 0 & 0 & 0 & 0 \\
0 & 0 & 0.121 & -0.512 & 0.256 & 0 & 0.366 & 0 & 0 \\
0 & -0.823 & 0 & 0.256 & 0.151 & 0.434 & 0 & 0 & 0 \\
0 & 0 & 0 & 0 & 0.434 & 0.568 & 0.193 & 0.346 & 0 \\
0 & 0 & 0 & 0.366 & 0 & 0.193 & -0.220 & 0.793 & 0 \\
0 & 0 & 0 & 0 & 0 & 0.346 & 0.793 & 0.076 & 0.899 \\
0 & 0 & 0 & 0 & 0 & 0 & 0 & 0.899 & 0 \\
0 & 0.899 & 0 & 0 & 0 & 0 & 0 & 0 & 0 \\
0.899 & 0.076 & 0.498 & 0 & -0.708 & 0 & 0 & 0 & 0 \\
0 & 0.498 & -0.018 & 0.098 & 0 & 0 & 0 & 0 & 0 \\
0 & 0 & 0.098 & -0.916 & 0.242 & 0 & -0.161 & 0 & 0 \\
0 & -0.708 & 0 & 0.242 & 0.078 & 0.666 & 0 & 0 & 0 \\
0 & 0 & 0 & 0 & 0.666 & 0.145 & 0.473 & 0.262 & 0 \\
0 & 0 & 0 & -0.161 & 0 & 0.473 & -0.264 & 0.824 & 0 \\
0 & 0 & 0 & 0 & 0 & 0.262 & 0.824 & 0.076 & 0.899 \\
0 & 0 & 0 & 0 & 0 & 0 & 0 & 0.899 & 0 \\
0 & 0 & 0 & 0 & 0.595 & 0.058 & 0.511 & 0.319 & 0 \\
0 & 0.899 & 0 & 0 & 0 & 0 & 0 & 0 & 0 \\
0.899 & 0.076 & 0.570 & 0 & -0.651 & 0 & 0 & 0 & 0 \\
0 & 0.570 & -0.292 & 0.442 & 0 & 0 & 0 & 0 & 0 \\
0 & 0 & 0.442 & -0.685 & 0.153 & 0 & 0.072 & 0 & 0 \\
0 & -0.651 & 0 & 0.153 & 0.305 & 0.595 & 0 & 0 & 0 \\
0 & 0 & 0 & 0 & 0 & 0 & 0 & 0.899 & 0
\end{array}\right)
$$


The filter could be constructed with dual-mode resonators (cavities), however this requires a complex coupling system, such as offsetting coupling and resonator elements [28], for controlling both couplings $M_{57}$ and $M_{67}$. The filter is therefore chosen to be implemented using mono-mode rectangular cavities as shown in Figure 9. The structure consists in two stacked blocks, each block gathering several cavities and being separated by a metallic plate with several coupling apertures. All cavities are excited on their $T E_{111}$ mode, excepting the sixth cavity, which is excited on its $T E_{112}$ mode for facilitating the coupling with both cavities 5 and 7 . Rectangular windows couple the cavities within each block, whereas rectangular or circular apertures are used in the metallic plate for realizing either a magnetic or an electric coupling.

The computer-aided design is performed using an electromagnetic model of the filter. A preliminary dimensioning stage, using simplified structures, is applied for initializing respectively, the width of each cavity, the width of each coupling window and the width or the radius of each coupling aperture, with respect to the ideal coupling parameters specified in (4). The dimensions of the electromagnetic model are then adjusted more precisely, identifying, at each step, the proper coupling parameters from the exhaustive set of solutions as explained in the previous section. One can note that during tuning iterations, the number of extracted coupling matrices fluctuates since the number of real solutions depends on the coefficients of the characteristic polynomials.

The hardware prototype is also tuned using coupling parameters extraction for adjusting tuning screws in each cavity and coupling window. The scattering parameters obtained from the electromagnetic model and from the hardware prototype are compared in Figure 10.

\section{Conclusion}

This article presents general techniques for the synthesis and the design of coupled resonator filters. Following the natural steps of microwave filter design, the first part deals with the synthesis of the low-pass prototype circuit and the second part with the dimensioning of the microwave distributed filter.

The synthesis of the prototype circuit focuses on the compatibility between the coupling topology and the filtering function to be realized, providing some guidelines in order to select a proper coupling topology and be able to solve the coupling matrix synthesis problem. The dimensioning of the distributed filter is centered on parameter extraction techniques. A particular attention is paid on the computer-aided design and tuning of filters whose coupling matrix synthesis problem admits several solutions. Finally, an illustrative example is taken of a microwave filter with a $(7,3)$ asymmetric characteristic realized with a generalized extended box topology.

\section{References}

[1] R.V. Snyder, Practical Aspects of Microwave Filter Development, IEEE Microwave Magazine, Volume 8 , Issue 2, pp 42-54, April 2007

[2] C. Kudsia, R. Cameron, W.C. Tang, Innovation in Microwave Filters and Multiplexing Networks for Communications Satellite Systems, IEEE Transactions on Microwave Theory and Techniques, Volume 40, Issue 6, pp 1133-1149, June 1992

[3] R. Levy, R.V. Snyder, G.L. Matthaei, Design of Microwave Filters, IEEE Transactions on Microwave Theory and Techniques, Volume 50, Issue 3, pp 783-793, March 2002

[4] G.L. Matthaei, L. Young, and E.M.T. Jones, Microwave Filters, Impedance-Matching Networks and Coupling Structures, New York: McGraw-Hill, 1964.

[5] D. Swanson,G. Macchiarella, Microwave filter design by synthesis and optimization, IEEE Microwave Magazine, Volume 8, Issue 2, pp 55-69, April 2007

[6] H.C. Bell, The Coupling Matrix in Low-Pass Prototype Filters, IEEE Microwave Magazine, Volume 8, Issue 2, pp 70-76, April 2007

[7] A.E. Atia, A.E. Williams, Narrow-Bandpass Waveguide Filters, IEEE Transactions on Microwave Theory and Techniques, Volume 20, Issue 4, pp 258-265, April 1972

[8] R. Cameron, Synthesis of Advanced Microwave Filters Without Diagonal Cross-Couplings, IEEE Transaction on Microwave Theory and technique, Volume 50, Issue 12, pp 2862-2871, December 2002 [9] R. Cameron, General Coupling Matrix Synthesis Methods for Chebyshev Filtering Functions, IEEE Transactions on Microwave Theory and Techniques, Volume 47, Issue 4, pp 433-442, April 1999

[10] S. Amari, Synthesis of Cross-Coupled Resonator Filters Using an Analytical Gradient-Based Optimization Technique, IEEE Transaction on Microwave Theory and technique, Volume 48, Issue 9, pp 1559-1564, September 2000

[11] M. Yu \& al., Predistortion Technique for Cross-Coupled Filters and Its Application to Satellite Communication Systems, IEEE Transaction on Microwave Theory and technique, Volume 51, Issue 12, pp 25052515, December 2003

[12] V. Lunot, S. Bila, F. Seyfert, Optimal Synthesis for Multi-band Microwave Filters, 2007 IEEE MTT-S International Microwave Symposium Digest, June 2007 
[13] Tutorial: “From S to M", http://www-sop.inria.fr/apics/Dedale/Doc/S2M.html

[14] H.C Bell, Canonical asymmetric coupled-resonator filters, IEEE Transaction on Microwave Theory and technique, Volume 30, pp 1335-1340, September 1982

[15] S. Amari, On the Maximum Number of Finite Transmission Zeros of Coupled Resonator Filters with a Given Topology, IEEE Microwave and Guided Wave Letters, Volume 9, Issue 9, pp 354-356, Sept. 1999

[16] R. Cameron, J.C. Faugère, F. Roullier, F. Seyfert, Exhaustive Approach to the Coupling Matrix Synthesis Problem and Application to the Design of High Degree Asymmetric Filters, International Journal of RF and Microwave Computer Aided Engineering, Volume 17, Issue 1, pp 4-12, Jan. 2007

[17] W.A Atia, K.A Zaki, and A.E Atia, Synthesis of general topology multiple coupled resonators filters by optimization, IEEE MTT-S Int. Symp. Digest, Volume 2, pp 1693-1698, September 1998

[18] S. Tamiazzo, G. Macchiarella, An Analytical Technique for the Synthesis of Cascaded N-Tuplets CrossCoupled Resonators Microwave Filters Using Matrix Rotations, IEEE Transactions on Microwave Theory and Techniques, Volume 53, Issue 5, pp 1693-1698, May 2005

[19] http://www.sop-inria.fr/apics/Dedale

[20] P. Lenoir, S.Bila, F.Seyfert, D.Baillargeat, S.Verdeyme, Synthesis of Assymetrical Dual-band Bandpass Filter based on Equivalent Network Simplification, IEEE Transaction on Microwave Theory and technique, Volume 54, Issue 7, pp 3090-3097, July 2006

[21] H.L. Thal, Computer-Aided Filter Alignment and Diagnosis, IEEE Transactions on Microwave Theory and Techniques, Volume 26, Issue 12, pp 958-963, Dec. 1978

[22] A. Garcia-Lamperez, S. Llorente-Romano, M. Salazar-Palma, T.K. Sarkar, Efficient Electromagnetic Optimization of Microwave Filters and Multiplexers Using Rational Models, IEEE Transactions on Microwave Theory and Techniques, Volume 52, Issue 2, pp 508-521, Feb. 2004

[23] P. Kozakowski, M. Mrozowski, Quadratic Programming Approach to Coupled Resonator Filter CAD, IEEE Transactions on Microwave Theory and Techniques, Volume 54, Issue 11, pp 3906-3913, Nov. 2006 [24] S. Bila, D. Baillargeat, M. Aubourg, S. Verdeyme, P. Guillon, F. Seyfert, J. Grimm, L. Baratchart, C. Zanchi, J. Sombrin, Direct Electromagnetic Optimization of Microwave Filters, IEEE Microwave Magazine, Volume 2, Issue 1, pp 46-51, March 2001

[25] P. Harscher, R. Vahldieck, and S. Amari, Automated Filter Tuning Using Generalized Low-pass Prototype Networks and Gradient-based Parameter Extraction, IEEE Transactions on Microwave Theory and Techniques, Volume 49, Issue 12, pp. 2532-2538, Dec. 2001.

[26] F. Seyfert, L. Baratchart, J.P. Marmorat, S. Bila, J. Sombrin, Extraction of Coupling Parameters For Microwave Filters: Determination of a Stable Rational -Model from Scattering Data, 2003 IEEE MTT-S International Microwave Symposium Digest, June 2003

[27] F.T. Hsu, Z. Zhang, K.A. Zaki, A.E. Atia, Parameter Extraction for Symmetric Coupled-Resonator Filters, IEEE Transactions on Microwave Theory and Techniques, Volume 50, Issue 12, pp 2971-2978, Dec. 2002

[28] R.J. Cameron, Dual-Mode Realisation for Asymmetric Filter Characteristics, ESA Journal, Volume 6, Issue 3 , pp 339-356, 1982

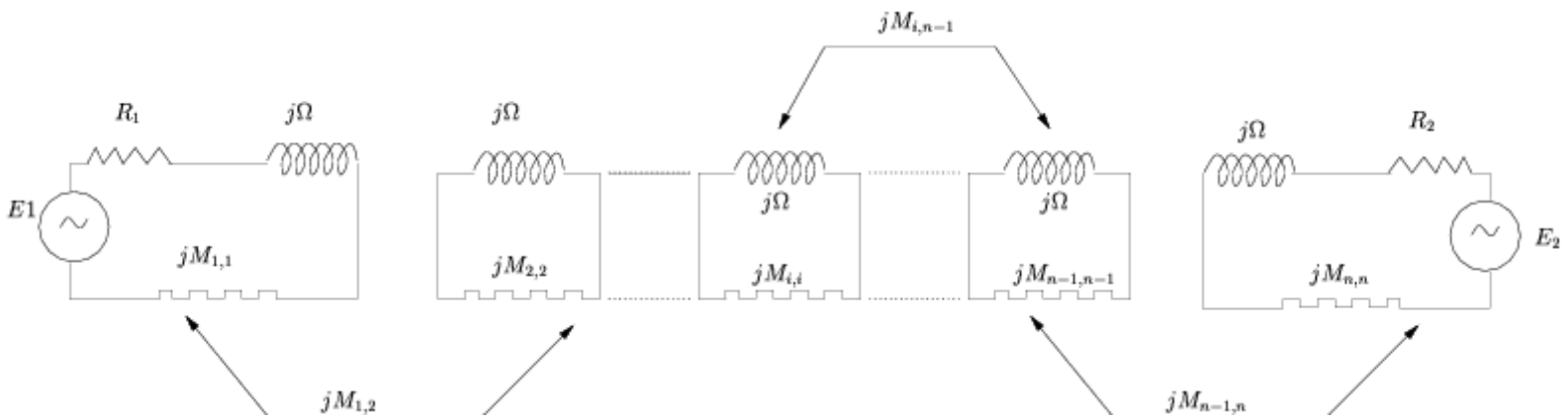

Figure 1: Low pass circuit prototype, where $\mathrm{j} \Omega$ symbolizes a unit inductance 


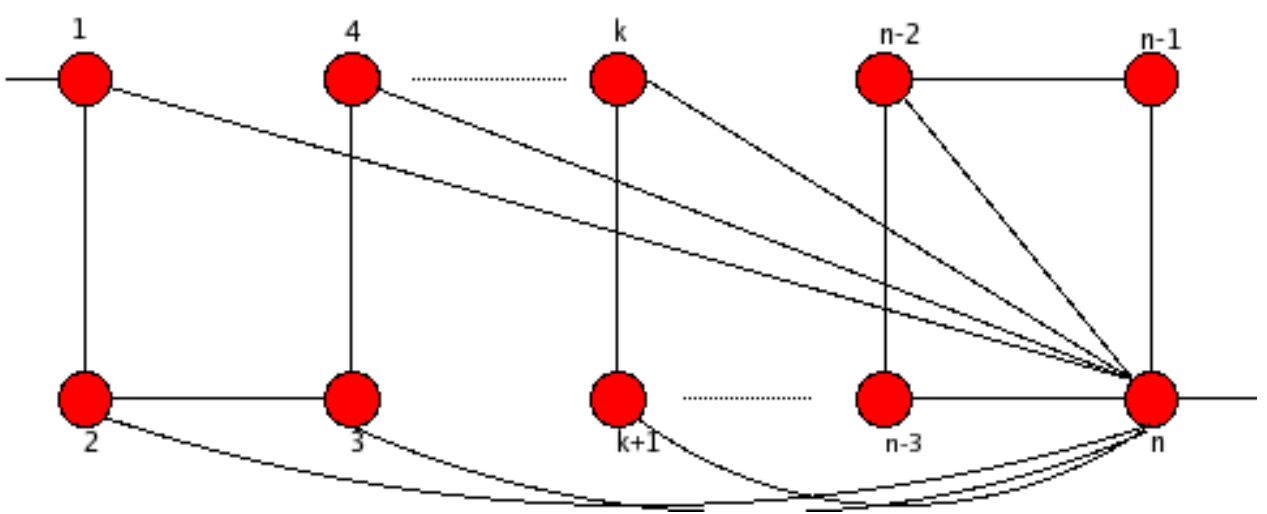

Figure 2: General "arrow" form
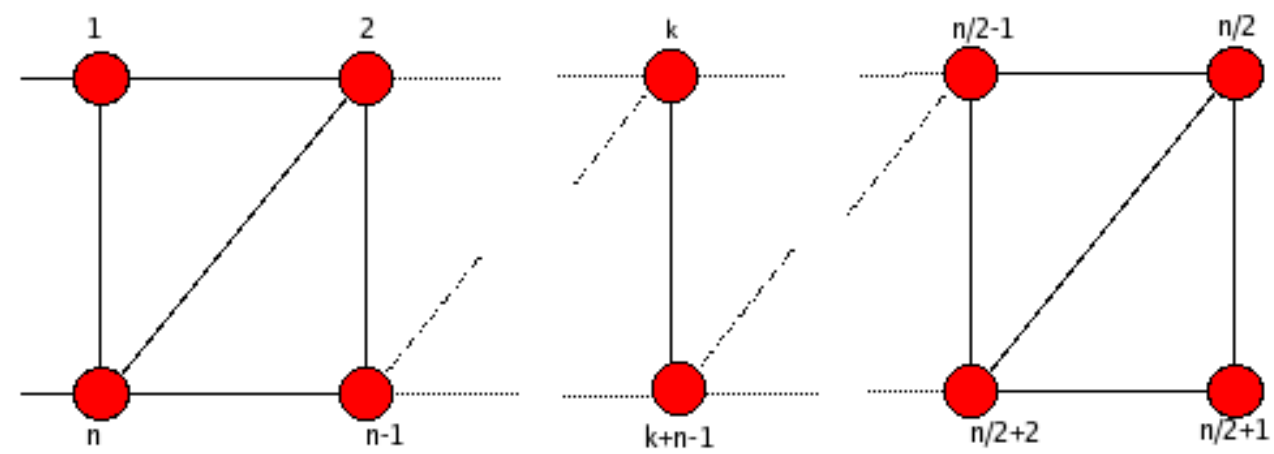

Figure 3: General folded form

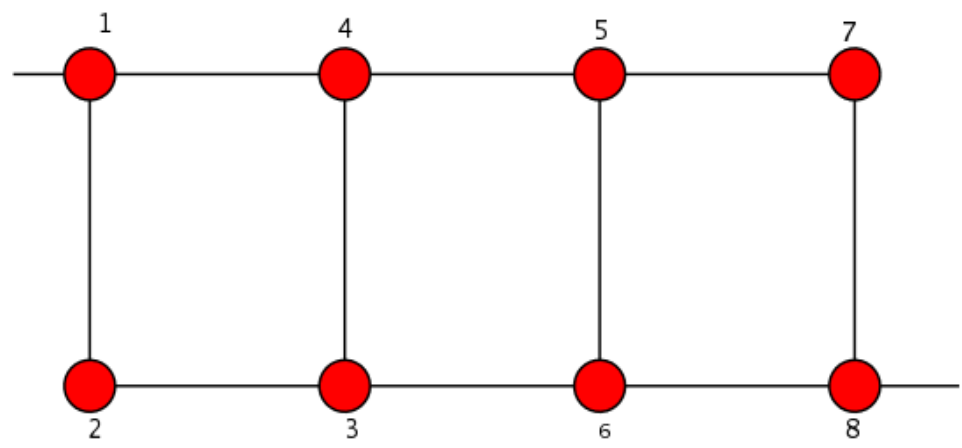

Figure 4: 8th degree extended box topology 


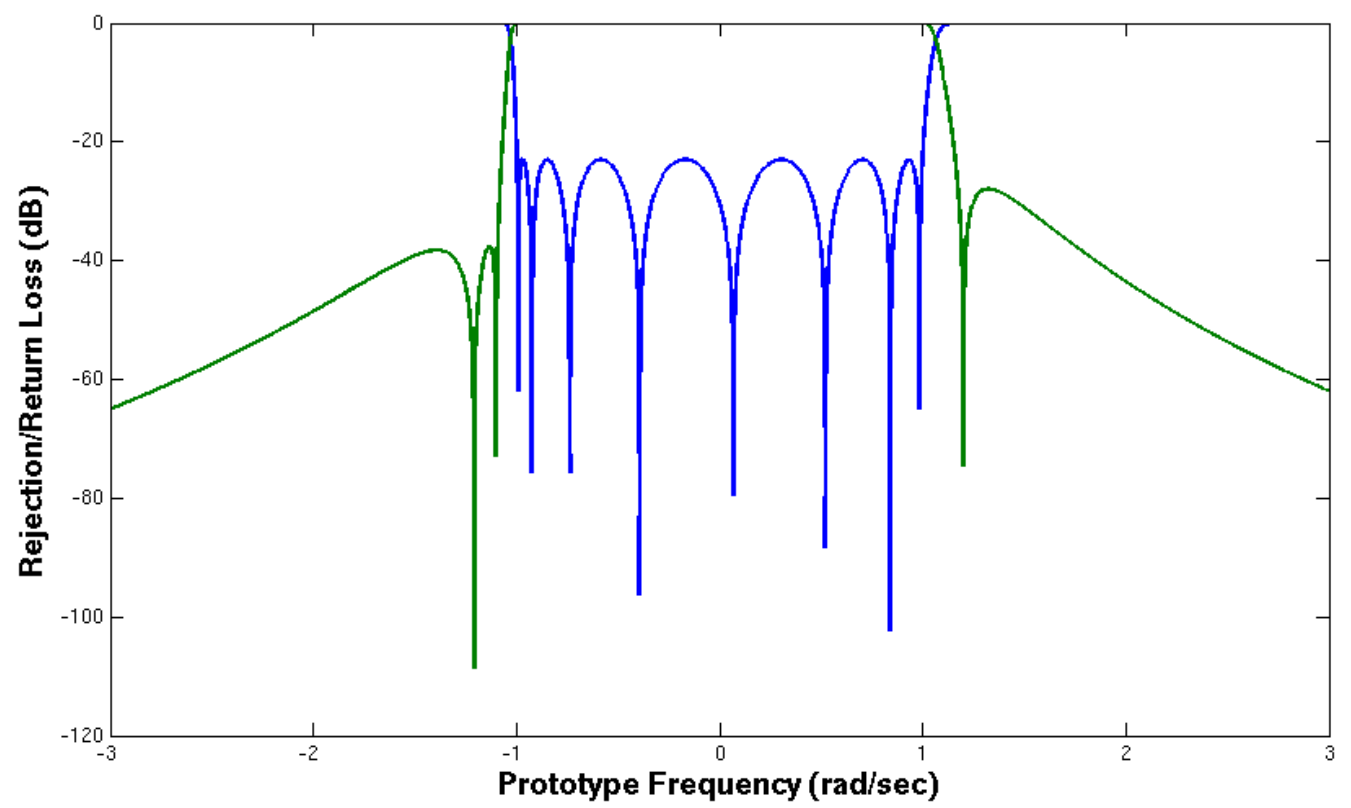

Figure 5: $(8,3)$ asymmetric filtering characteristic

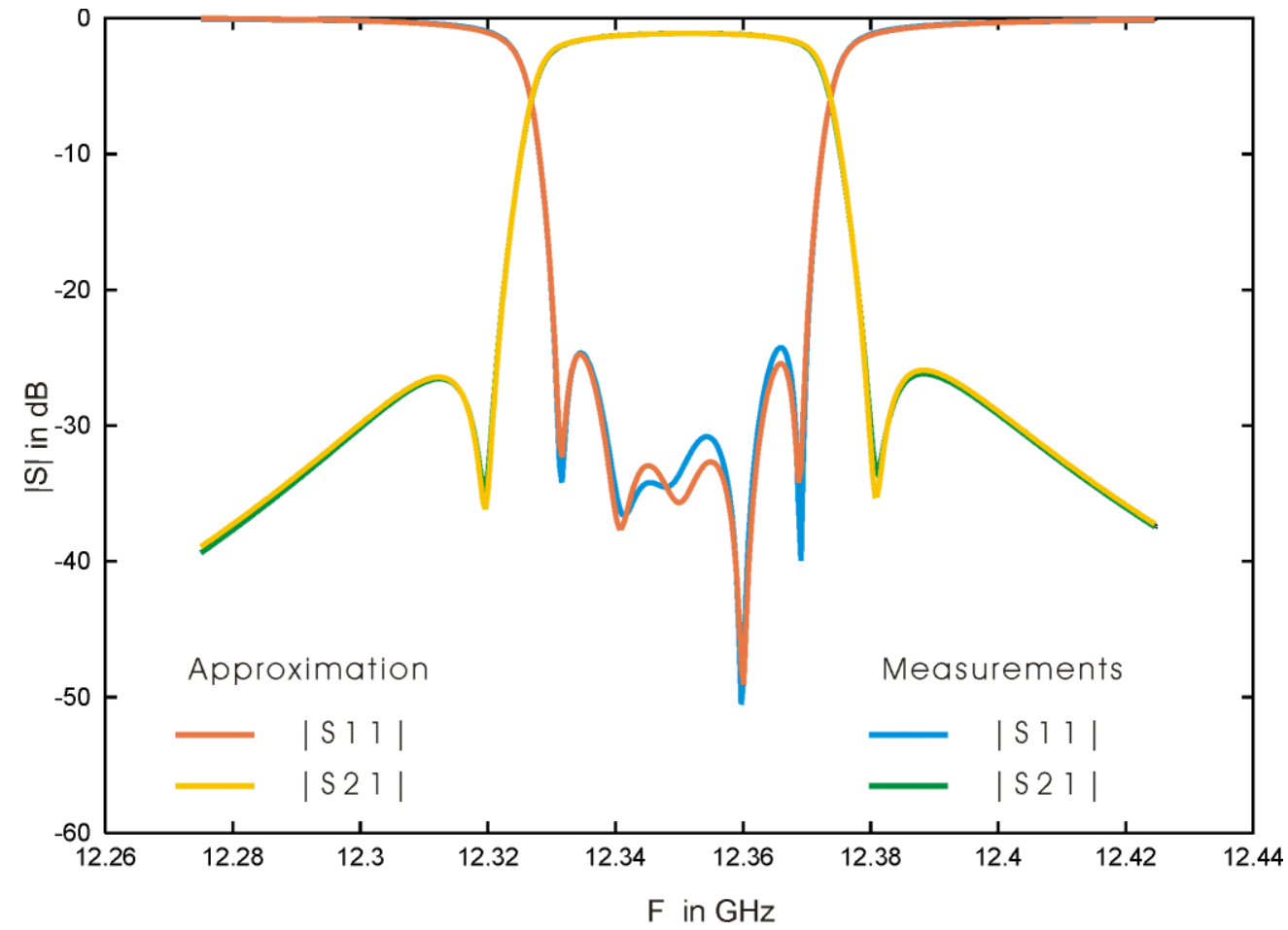




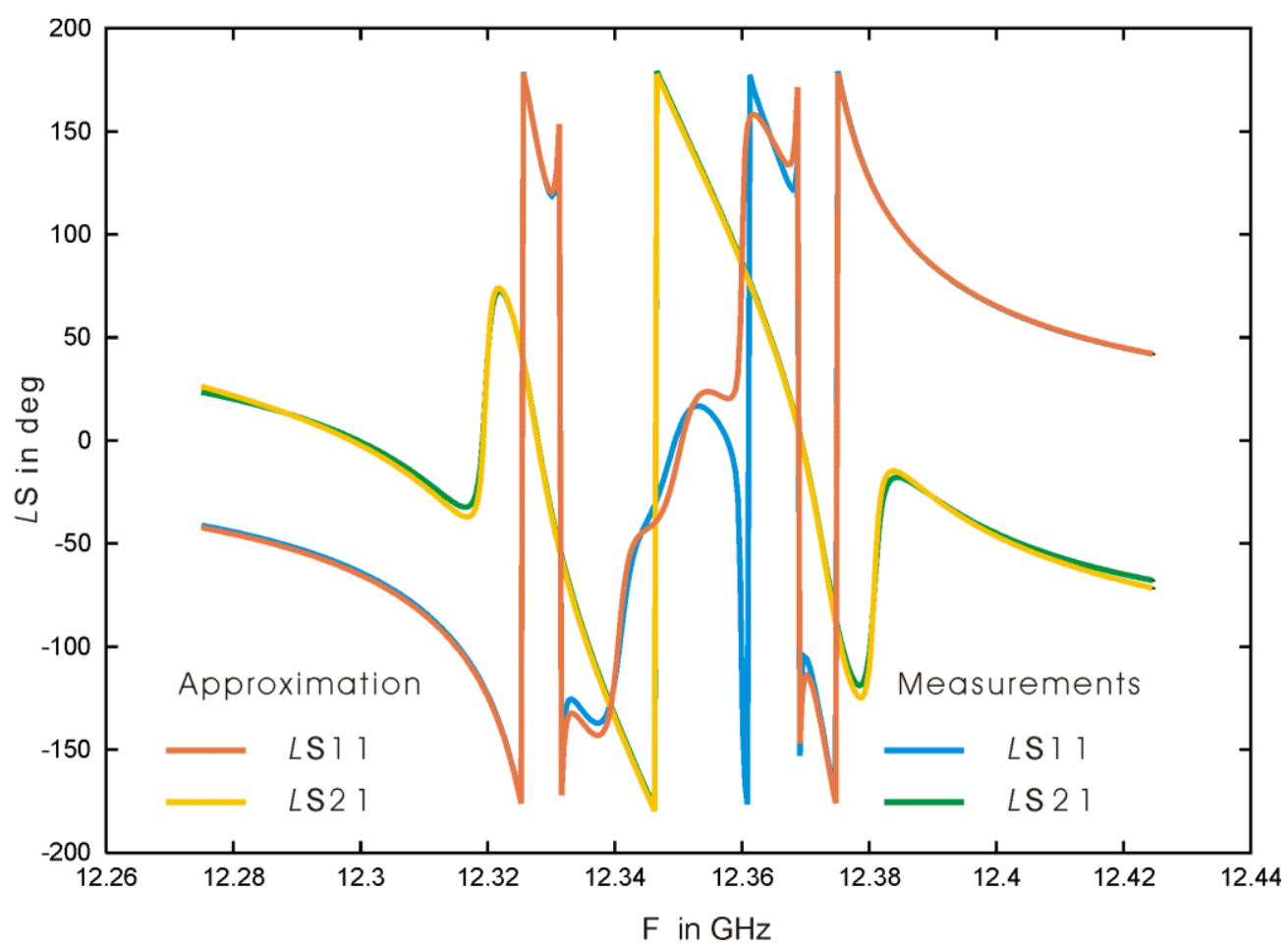

Figure 6: Rational approximation of measured scattering parameters

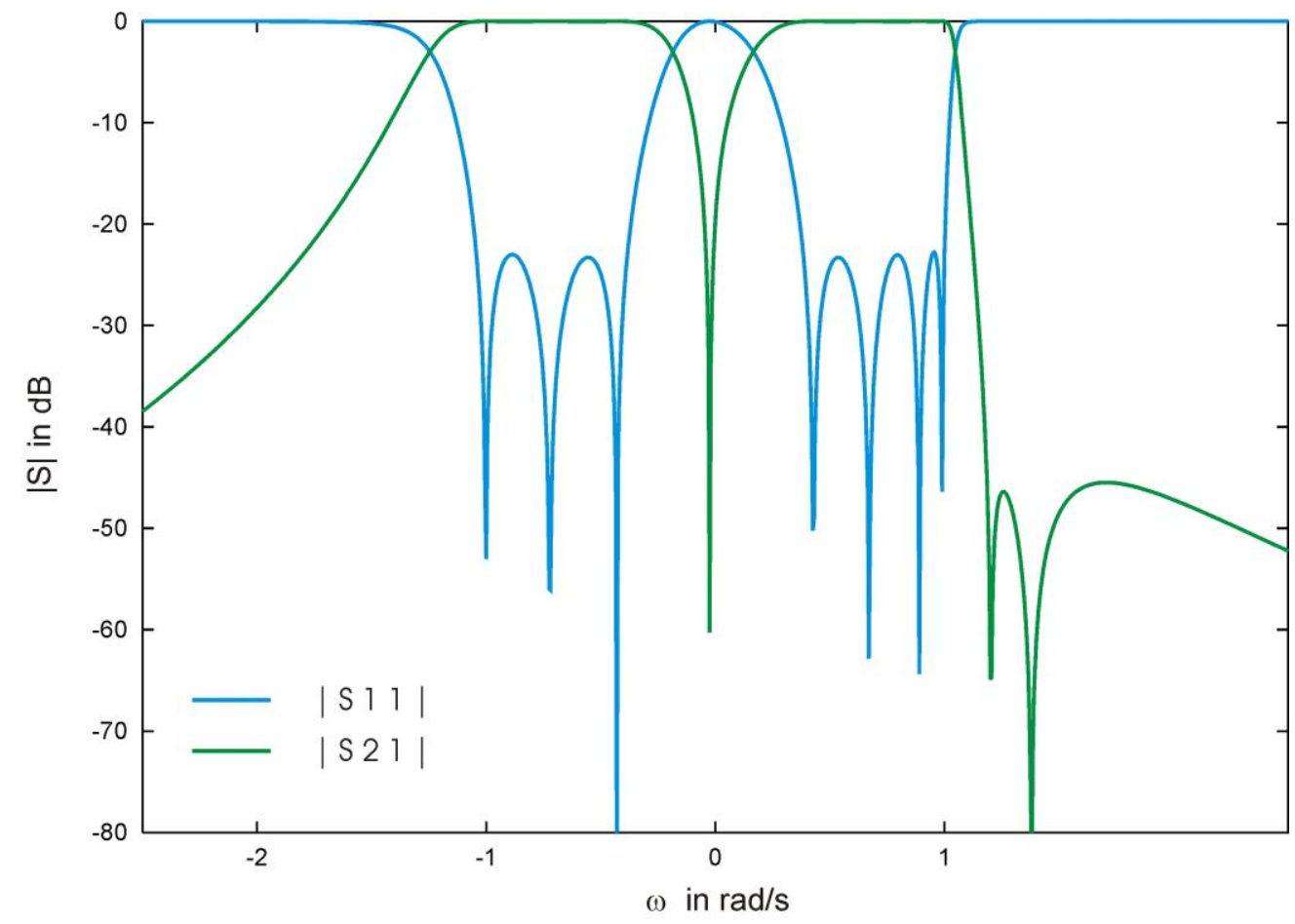

Figure 7: Normalized (low-pass) scattering parameters (7 pole 3 zero asymmetrical characteristic) 


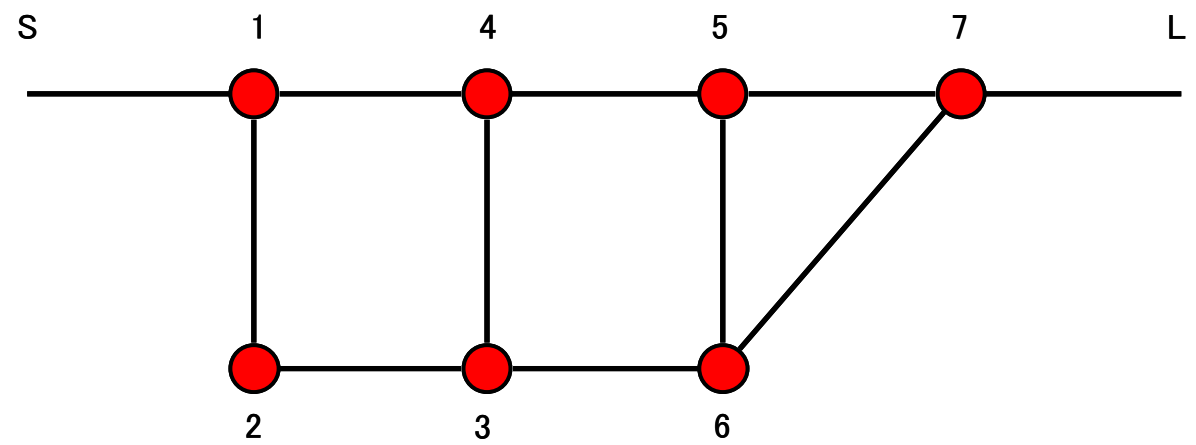

Figure 8: Coupled resonator network: generalized extended box providing a 7 pole 3 zero asymmetrical characteristic

First block: cavities \# 1, 4, 5 and 7

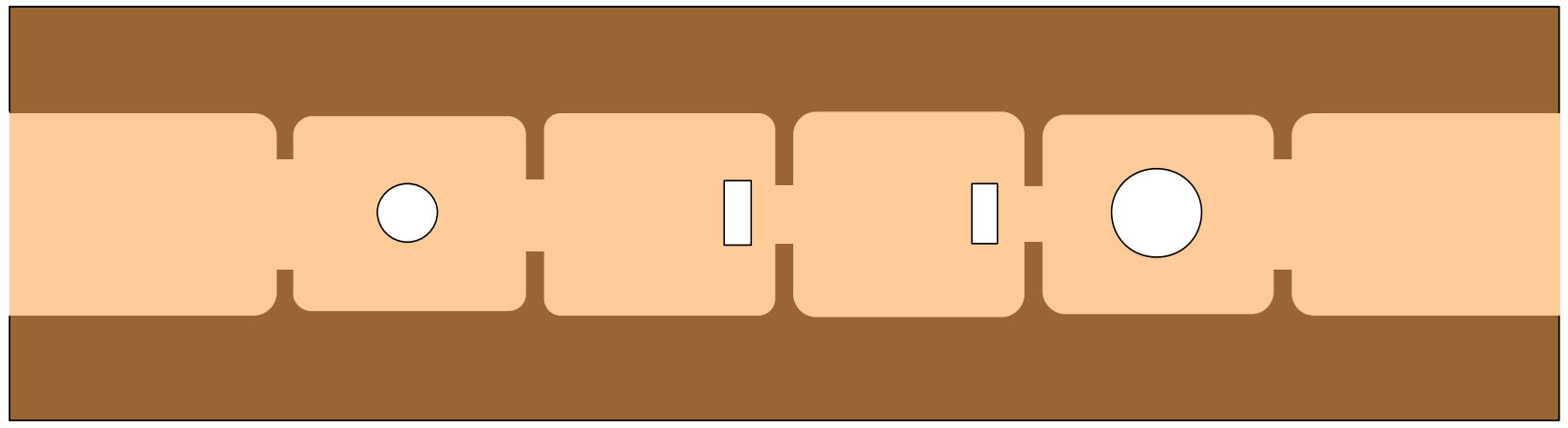

Second block: cavities \# 2, 3 and 6

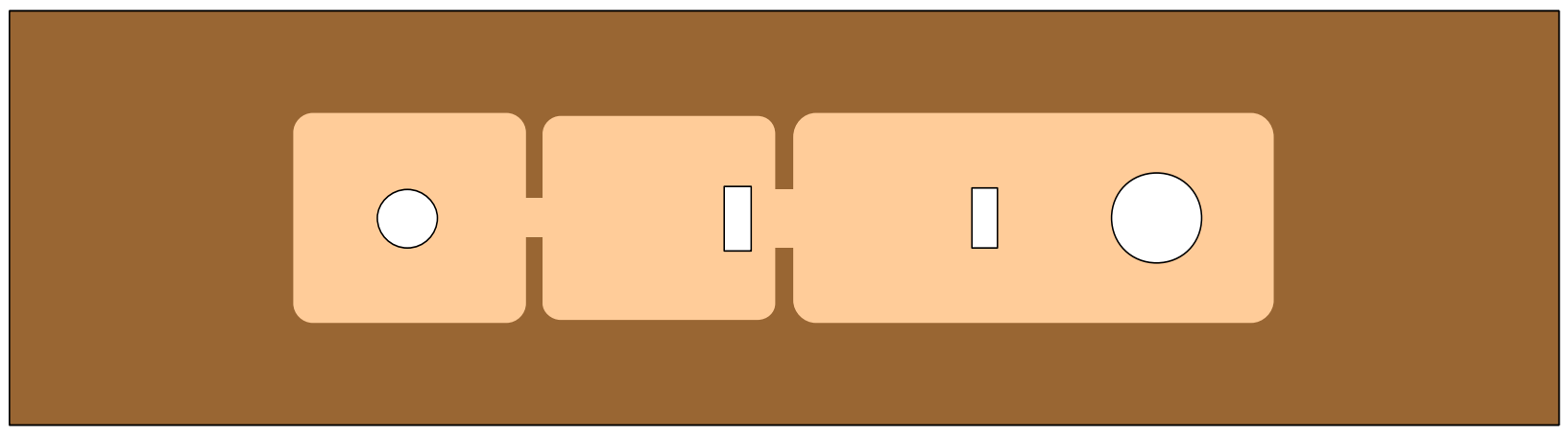

Figure 9: 7 pole 3 zero dual-band filter implemented with stacked mono-mode rectangular cavities 


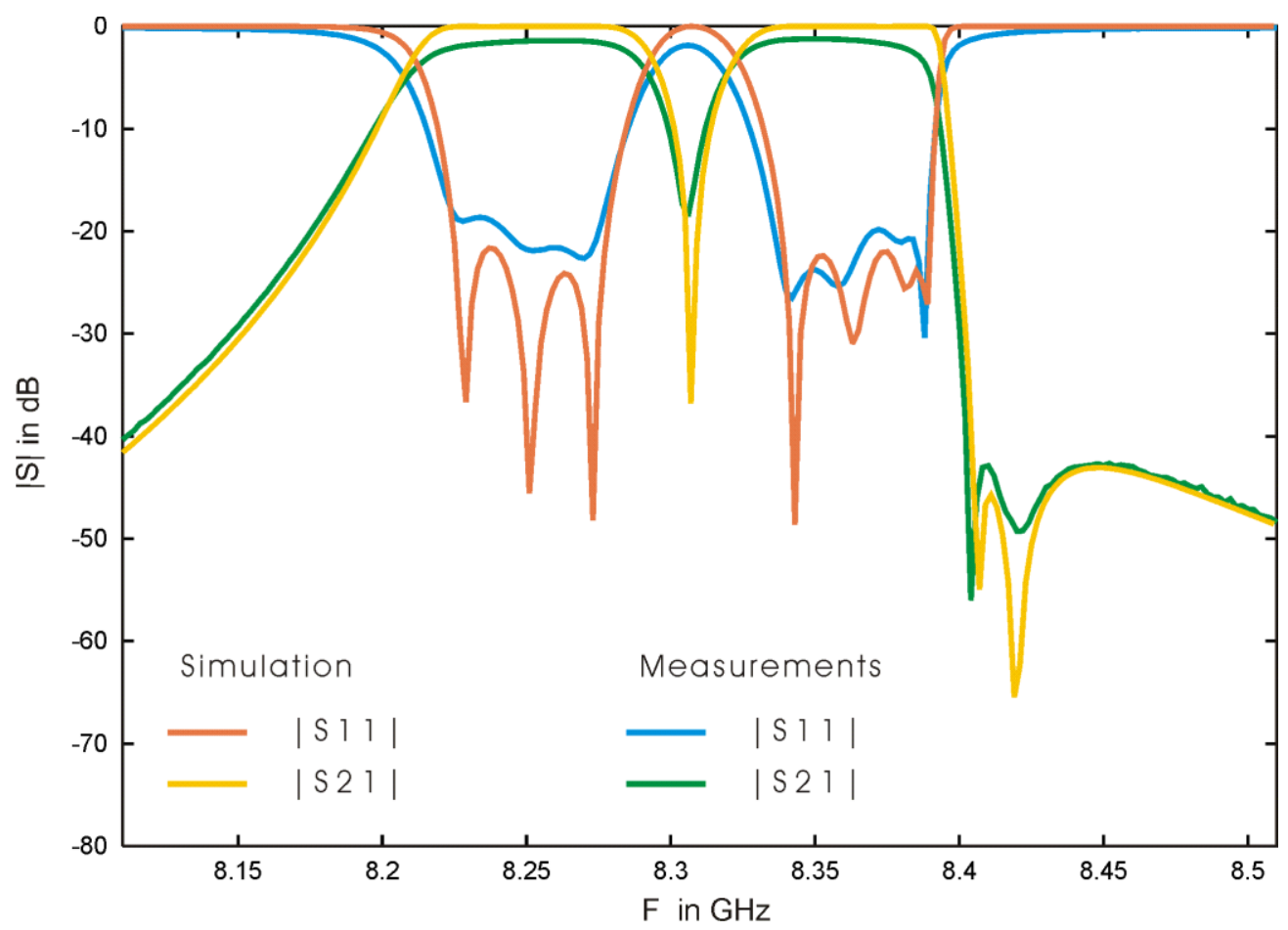

Figure 10: Simulated and measured scattering parameters of the 7 pole 3 zero dual-band filter 